\title{
Reflections on the Development of Contemporary Chinses Folk Art
}

\author{
Jian Gao
}

\begin{abstract}
Hunan Normal University, Yuelu District, Changsha, Hunan, China
Corresponding Email: liu_sai@hnu.edu.cn
\end{abstract}

\begin{abstract}
Folk art is a reflection of folk social life, which not only carries rich history and culture, but also reflects the cultural features of folk beings, as well as the spiritual characteristics and aesthetic taste of a nation. Folk art has rich research value, which also provides the possibility for folk art scholars to study from different angles. This study analyzes the characteristics and problems of Chinese folk art in modern society.
\end{abstract}

Keywords: Folk art, Contemporary, Reflections

\section{对中国当代民间美术发展的思考}

\author{
高健
}

湖南师范大学, 岳麓区, 长沙, 湖南, 中国

liu_sai@hnu.edu.cn

\section{摘要}

民间美术是对民间社会生活的映射，既承载着丰富的历史文化，也体现了民间众生的文化风貌，同时反映了一 个民族的精神特质和审美情趣。民间美术有着丰富的研究价值, 这也为民艺学学者从不同的角度研究民间美术 提供了可能。本文针对中国民间艺术在现代社会呈现的特征及出现的问题进行分析。

关键词：民间美术，当代，思考

\section{1.概念及背景}

“民间”一词有两重含义，一层是非官方的地方， 第二层含义是在人民中。从 20 世纪开始“民间”概念 开始在中国流变, 在二十世纪三十年代以前的时候, “民间”就已成为各领域知识分子关注和讨论的焦点， 他们对民间这一概念达成共识，认为“民间”从地域空 间上是指农村地区，就文化的承载者而言主要是以农 民为主。对“民间”的关注与“五四”新文化运动时期民 俗学科最开始兴起的时候以及当时的知识分子充满 浪漫主义情怀有关。最初的民间艺术研究发端于民俗 学研究, 主要偏重对民间神话、民间歌谣的探索研究, 而之后就慢慢开始拓展到民俗习惯及信仰方面。但是 在这一时期还未明确提出“民间美术”这一概念。

在中国的二十世纪四十年代的延安地带, 民间艺
术因为革命背景时期的需要就展现出来一幅全新的 面貌, 在 1942 年的时候, 毛泽东发表了《在延安文 艺座谈会的讲话》这一文件, 提出相关的生产活动要 到民间去发展，要积极的向人民群众和优秀的民间文 化艺术学习的文艺方针 ${ }^{\circledR}$ 。当时的艺术工作者都在学 习民间艺术，例如剪纸、扎纸以及年画等一系列民间 艺术基础上进行了大量的创作活动。但是直至改革开 放前期, “民间艺术”仍然不是作为一个学术意义的概 念而被广泛的研究与探讨。

在改革开放后，国内开始掀起新一轮的民间艺术 研究的“热潮”。这与当时的人们的寻根文化意识以及 现代乡土情怀是分不开的，同时这也是对受到当时西 方文化以及西方艺术文化冲击的一种回应。当时许多 艺术家从民间艺术中汲取营养，从而使自己的创作更 具有乡土气息和民族风格。在二十世纪八十年代初的 
中国，“民间美术”终于作为学术意义的概念被提出来， 这就意味着对民间艺术的研究不再只是停留在对民 间工艺品的收集以及艺术家对其学习、借鉴的浅显层 次上，而是需要构建一个知识体系的构架。

构建民间美术历史与理论知识体系，这本身就可 以成为往后学术研究反思的对象。使得后来的学者得 以来考证“民间美术”概念的形成与发展过程。在这一 过程中有一位民间美术界重要的奠基人一一张道一 先生，他对于“民间美术”的认识以及后来研究“民间 美术”的学者们产生了极其深刻的影响。张道一先生 提出了“民间美术”的年画花纸、门神纸马、剪纸皮影、 陶瓷器血、雕刻彩塑、印染织绣、编结扎作、儿童玩 具和其它等九个类别。张道一先生是中国国内引介日 本民艺家柳宗悦先生所倡导的“民艺学”的最早的学 者, 并提出建立独立的“中国民艺学” (2。张道一先生 认为“美术包括了工艺, 但民间美术的主流, 不论就其 量来说还是就其面来说, 则是以工艺为主”。也许因为 这个判断“民间美术”提法逐渐被舍弃掉了，现在更多 场合使用的是“民艺学”的概念。

中国民族美术分为宫廷美术、文人士大夫美术、 民间美术, 其中民间美术的存在形式最广泛最为普遍 的。民间美术以其简单的造型, 充分满足了人民生活 的装饰特征和实用的功能性, 从物质以及精神上满足 了底层劳动人民的需求。劳动人民创造了满足他们自 己对于生活的期待与希望的民间美术，反过来说民间 美术也反映了劳动人民的生产生活、生活习惯、理想 情感等等。从广义上来说, 民间艺术包括工艺美术、 戏曲、民间音乐等多种形式; 从狭义上来说, 民间艺 术是指民间造型艺术，民间美术和工艺美术各种表现 形式。从某种意义上来讲，民间美术是民间艺术的一 部分, 它们是紧密联系相辅相成的存在。

\section{2. 产生及特点}

本文为了更好地分析探究目前中国民间美术的 发展进程, 则必须对其的特征进行分析。民间美术的 独特的特征使得它有别于其他艺术形态, 它独有的艺 术存在形式、以及具有深厚的文化内涵都代表了中华 民族的民族审美历程, 笔者借鉴众学者的观点, 将其 基本特征总结归纳为以下几点:

\section{1 乡土性}

民间美术的创造者大都是生活在社会底层的劳 苦大众, 他们在生产生活、劳动实践的过程所产生的 事迹以及生长的背景环境, 使得他们对生活的感受直 接激发了他们的创造力, 劳动人民既是创造者又是使 用者, 同时还是民间美术的欣赏者。三重身份重叠使 得他们的作品更能让观赏者感受到他们的情绪延伸。 在劳动生产的过程当中, 他们会自由地按照自己的意 趣与想象进行创造, 这种来源于生活的艺术创造充满 着浓郁的乡土气息, 具有独特的乡土性。因此朴实大 方、自然稚拙是它们的一般特征, 民间美术往往体现
了当时人们真情实感的流露, 这使得它具有一种不同 于其他艺术形式的魅力。

\section{2 地域性}

中华大地地域辽阔，地形复杂，民族众多，这使 得个地域之间人们生活环境、成长背景、风俗习惯有 着很大的不同。不仅南北有差异, 即使同是南方城市 或同是北方城市，也会有一定的差异性。因此这就决 定了民间美术具有地域性的特征。例如同样是家具形 式，却有着京式、广式、苏式等地方性的区别。刺绣 工艺同样也体现了这一特征, 刺绣工艺因为地域不同 而有不同种类区别，例如蜀绣、湘绣、京绣、苏绣、 粤绣等等; 因为不同民族有着不一样的的信仰，体现 在工艺美术上会有造型偏好不同、颜色倾向不同、材 质喜好不同等等, 例如苗族的蜡染, 土家族织锦等。 不同的信仰会民间美术导致不同的主题、色彩、制作 方式等等。这些因素都是都决定着民间美术的地域性 差别，所以民间美术具有地域性的不同。

\section{3 群体性}

中国民间美术大都是来自于广大人民群众的生 活艺术, 它的存在形式、繁多种类都来源于人民的生 活与劳动当中, 具有着非常强大的生命力。这种生命 力不仅仅是因为于人群的广大, 更因为它拥有的是绵 延不断、代代相传的时间延续性。在代代口耳相传的 过程当中，人民群众的民间美术不断地进行着创新、 丰富，因此民间美术在不同的时代有着不同的时代特 色。但是在这变化当中，民间美术的主题与色彩、形 式与内容又具有一定的程式化和一定的规范化要求。

\section{4 实用性}

民间美术另外一个重要的特征就是实用性。在劳 动人民生产生活、衣食住行、风俗习惯、信仰追求中 都有着民间美术出现的影子。在进行民间美术创造的 过程当中, 人们使用的材料或者材质大都是生活中随 处可见的材料, 例如剪纸所用的纸张, 皮影所需的动 物皮毛, 刺绣所需要的棉麻丝轻等等。人们在创作的 时候, 首先考虑的是物件的实用性, 即好用以及耐用, 其次才是考虑它的美观性, 是否好看以及耐看。因为 创作者大都是底层的劳动人民, 在封建社会中, 生存 就已经是一件不太容易的事情, 所以他们在制作工具

(创造艺术品) 的时候, 会极尽所能地通过仅有的材 料来进行装饰。更多的体现出来的便是材料本省所具 有的肌理、光泽、纹饰、硬度等非常原始自然的特征。

\section{5 寓意性}

民间美术是生活在社会最底层的劳动人们所创 造的, 他们所受到的阶级压迫是最重的, 他们曾经或 多或少都饱受过现实摧残压制, 但是反映这些在人们 生产、创造的民间美术上, 我们看到的几乎全都是具 
有吉庆的、表达美好的寓意的物件。这种坚韧、乐观 的精神通过一件一物的制作与传授间也在传承、哺育 着深沉的中华民族精神。这也启示着现代我们在学习、 研究传统的民间美术时不要止步于表象吉庆元素的 搬移挪用，而应当去了解民间美术文化的内核。

中国民间美术是中国劳动人民在中华文化孕育 中所不断发展形成的, 它体现了劳动人民在他们的时 代里朴素的世界观、价值观、艺术观、民族观、乃至 哲学观, 即便他们在生产的时候并不自知。中国民间 美术从原始社会发展至近代, 它的使用群众最为广泛、 历史文化内涵最为丰富, 艺术形态最为自由。

\section{3. 分析归类}

笔者归纳了从二十世纪初至今, 中国众多学者曾 对民间美术进行过田园调查、实地考察、搜集资料、 整理分类等, 但是这些研究不够系统, 十分薄弱。将 民间美术分析归类不是简单地将其进行颜色、造型、 技法、区域、时代等分析归类, 而是要将这些所呈现 的表象类别研究转变为对民间美术进行风俗习惯、造 物态度、生活方式等的研究, 即从源头上进行分析研 究。张道一先生“为生活造福的艺术”的论断其实正是 建立在将民间美术视为民众的生活方式和文化方式 的基础上提出的 ${ }^{\circledR}$ 。

众多学者将民间美术从各个角度进行不同的类 别分析, 而笔者对中国民间美术进行了简单的归类分 析, 一共分为以下六个主要分类:

\section{1 起居类:}

人民群众居住的建筑物以及与人们生活有关的 其它建筑物品, 建筑物内的构成建筑物的砖、木、石 雕刻工艺品以及室内空间中的家具陈设等;

\section{2 生活类:}

人们平时所穿的服饰以及穿戴包括刺绣印染、民 族服饰、衣物鞋垫、绣花荷包、嫁衣、织锦、蜡染等 等;

\section{3 娱乐类:}

人们在戏曲中会使用到的皮影、脸谱、面具、木 偶以及与戏曲文化相关的小物品、以及民间玩具例如 拨鼓、风筝、糖人等;

\section{4 装饰类:}

主要包括人们生活中装点生活环境、以及自身的 的装饰品, 例如窗花剪纸、年画版画以及竹草编等;

\section{5 生产类:}

人们在生产活动中使用的一系列器具、包括农业
用具、交通工具以及使用的各种材质的饮食炊具、以 及其它生活用品等;

\section{6 祭祀类:}

与民俗活动中丧葬嫁娶、节日祝神、符道神像等 相关活动中会用到的供品、塑像、纸扎。

对民间美术的分类是相对的, 从人们的生活习俗 中进行性类别分析尽量避免将其碎片化、孤立化。通 过类别的分析，我们可以立体系统全面地了解民间美 术所呈现的文化形态。

\section{4. 现状}

民间美术作为人们精神与物质相结合的产物，民 间美术与人们生活交织在一起的，人们的生活环境、 价值观念、审美意趣都会影响到民间美术的发展变化。 当人们的生活条件、生活方式在发生改变后, 具有实 用性的民间艺术也会随之而产生改变, 否则会逐渐呈 现颓势, 甚至消失。

民间美术出现改变不仅在数量上, 也表现在质量 上。例如民间美术中的窗花剪纸，作为老百姓所喜闻 乐见的艺术形式, 在过去十分受欢迎, 在婚宴喜事、 过年佳节时经常会被用到, 但是在现代人们很少剪窗 花装饰自己的窗户，这种艺术更多作为传统文化被保 护起来, 或是作为传统符号被艺术家借鉴到自己的艺 术品中去, 而人们很少在生活中制作和使用它。随着 时代的发展, 现代的建筑中窗户都非常简洁敞亮, 极 少在玻璃上用窗花装饰。人们宁愿贴海报、照片以及 其它印刷品而非剪纸窗花来进行装饰。事实上, 不止 剪纸窗花这一种民间美术形式在出现颓势, 而是整体 上的民间美术受到了冷落。

造成民间美术呈现低谷状态的原因有很多方面, 一是社会生活与生产方式的快速发展, 在互联网和人 工智能时代与从前人们的小农经济男耕女织的时代 完全不同了, 民间美术最本质的特征是小农经济为其 生产基础。中国近三十年的发展速度飞快, 社会在加 速转型, 小农经济早已被取代, 人们不再是“自给自足” 生产的方式。也从农业生产时代转变成为工业化机器 生产方式, 生产生活的方式改变使得生活方式也发生 了改变, 传统的民间艺术在这个过程中逐渐呈现式微 的甚至走向衰落的状态。民间美术品在现代几乎都是 以商品的形式出现的, 例如以旅游产品或是文创产品 的形式出现。同时因为社会结构变化, 社会的主要阶 级不再是底层的劳苦民众, 更多的是中层阶级, 这巨 大的变化使得民间美术走向衰落成了事实。在现代快 速发展的时代里我们在不断地努力去保留民间美术， 保存和恢复他们的制作方法、制作特征等。

\section{5. 结论}

在机器化生产的大时代里, 人们仍然觉得手作带 给人亲近自然、接近传统的感觉从而比起流水线上的 
产品更加珍贵。这种珍贵不止在于物件, 更在于民间 艺术所传达给人的精神。一种对生活充满热切希望与 追求的精神, 一种满怀对未来充满渴望与美好的诉求, 这些的精神都体现在民间美术的一件一物之中。民间 美术不只在于一件一物的物质上, 更在于物件上所包 含的一个地域、一个民族的历史文化、审美意趣、伦 理宗教等等丰富的信息, 因此我们应当不止关注于如 何复兴某种手艺或是复原某件物什, 同时也应该关注 物件背后所蕴藏的精神。

人们曾认为传统已死, 我们花了很多精力去恢复 一些传统艺术品, 但是我们在生活当中已经很少会用 到了, 那它其实就已经失去了生命力。但其实传统民 间美术它可以被我们研究、运用, 再以新的形式语言 出现在我们现代的生活里, 而不再只是以一个单薄的 物件或是以一个符号的形式出现。例如剪纸窗花, 它 可以不再只是这一种形式, 可以被设计师理解吸收剪 纸的特点再将其运用进他们的标志设计、视觉设计、 包装设计、空间设计当中。在这种不断地吸收、运用、 再创造的过程中，传统的民间美术会以一种新的形态 “活着”。现在已经有着将民间美术向“文化创意品”、 “旅游文化艺术品”的“活态保护”的众多举措。中国台 湾“行政院文化建设委员会”在《文化白皮书》中对传 统文化和民族民间工艺所持的理念为: 一种文化如果 只有依赖先人的遗产自豪, 是不对的, 因为文化不仅 是固 (原) 有的部分而已, 它必须是一个有生命的活 的文化。因此只有让民间美术文化处于不断发展生长 的状态, 才是对它最好的传承与保护。人们对民间美 术的学习与将其文化演进还有这很长的路要走。

\section{REFERENCES}

[1] Qiao Xiaoguang. (2011). Chinese Folk Art. Hunan Fine Arts Publishing House, Changsha, China.

[2] Zhang Daoyi (1989). The Development of Chinese Folk Art. Beijing Arts and Crafts Publishing House, Beijing, China.

[3] Liu Min (2012). On the Theoretical Research of Folk Art since the 1980s, Master's Thesis, Shandong University of Art \& Design.

[4] Wu Xihong (2009). On the Contemporary Value of Children's Folk Art Education, Master's Thesis, Shandong Normal University.

[5] Sun Jianjie (2014). Reflections and cognition of contemporary Chinese folk art. Cultural Geography, 02:108-109.

[6] Wang Zhiqiang (2017). Cognition and Reflections on Contemporary Chinese Folk Art. Art Appreciation, 01: 114-115.
[7] Jin Yuli (2014). Cognition and Reflection on Contemporary Chinese Folk Art. Modern Communication, 07:80-81. 